\title{
Influence of skeletal muscle mass and fat mass on the metabolic and inflammatory profile in sarcopenic and non-sarcopenic overfat elderly
}

\author{
Fabrício E. Rossi ${ }^{1}$ - Fábio S. Lira ${ }^{2}$ Bruna S. A. Silva ${ }^{3} \cdot$ Ana Paula C. F. Freire $^{4} \cdot$ Ercy M. C. Ramos $^{4} \cdot$ Luis A. Gobbo $^{3}$
}

Received: 26 June 2018 / Accepted: 18 August 2018 / Published online: 3 September 2018

(c) Springer Nature Switzerland AG 2018

\begin{abstract}
Background Sarcopenic elderly present low muscle mass and strength, however, it is not clear if the inflammatory and metabolic profile is more related to low lean mass or high fat mass in sarcopenic and non-sarcopenic overfat elderly.

Aim To verify the difference in inflammatory and metabolic responses in sarcopenic and non-sarcopenic overfat elderly and the relationship between these markers, body composition, and strength in this population.

Methods Fifty-seven elderly were divided into two groups: sarcopenic $(n=30)$ and non-sarcopenic $(n=27)$. Body composition was evaluated with octopolar bioimpedance. Total cholesterol, high-density lipoprotein cholesterol, triacylglycerol, glucose, cortisol, leptin, adiponectin, Plasminogen activator inhibitor-1 (PAI-1), TNF- $\alpha$, IL-6, IL-8, and IL-10 were assessed. The handgrip test was used to evaluate strength.

Results When comparing the inflammatory profile, sarcopenic individuals showed greater adiponectin concentration $(p=0.019)$, adiponectin/fat mass ratio $(p<0.001)$, adiponectin/visceral fat $(p<0.001)$, and higher PAI-1 $(p=0.019)$ than non-sarcopenic overfat elderly. After adjusting the inflammatory profile by skeletal muscle mass the significant differences between groups were maintained $(p<0.05)$ but no significant differences between groups were observed when adjusting by fat mass, despite a tendency to a significant difference for adiponectin concentration $(p=0.06)$. In addition, after adjusting leptin by fat mass there was a statistically significant lower concentration in the sarcopenic compared to non-sarcopenic overfat elderly.

Conclusion Non-sarcopenic overfat elderly presented lower anti-inflammatory and anti-atherogenic responses than sarcopenic elderly. Furthermore, fat mass but not skeletal muscle mass seem to change these responses.
\end{abstract}

Keywords Sarcopenic $\cdot$ Inflammation $\cdot$ Elderly

Luis A. Gobbo

luisgobbo@fct.unesp.br

1 Immunometabolism of Skeletal Muscle and Exercise Research Group, Department of Physical Education, Federal University of Piauí (UFPI), Teresina, PI, Brazil

2 Exercise and Immunometabolism Research Group, School of Technology and Science, São Paulo State University (UNESP), Presidente Prudente, SP, Brazil

3 Laboratory of Skeletal Muscle Assessment (LABSIM), Post-graduation Program in Movement Sciences, School of Technology and Science, São Paulo State University (UNESP), Rua Roberto Simonsen, 305, CEP 19.060-900, Presidente Prudente, SP, Brazil

4 Department of Physiotherapy, School of Technology and Science, São Paulo State University (UNESP), Presidente Prudente, São Paulo, Brazil

\section{Introduction}

Aging, associated with high muscle mass loss can result in Sarcopenic syndrome [1], which leads to decreased muscle strength, and impaired locomotion and balance, thus increasing the number of falls due to frailty [2]. Low lean mass plus low muscle strength are predictors of mortality risk among elderly people [3]. Furthermore, muscle mass has an important role in inflammatory and metabolic response, given that cytokines such as IL-6 originate from muscle tissue (in exercise). Furthermore, IL-6 may have an anti-inflammatory effect by inhibiting TNF- $\alpha$ secretion [4], and increasing fat oxidation and glucose uptake via AMPK activation [5]. Not only this, but by activating muscle satellite cells, IL-6 initiates the hypertrophic process [6]. 
Several cross-sectional [7] and longitudinal studies [8, 9] have verified the relationship between muscle mass and inflammatory and metabolic markers. Aleman et al. [8] conducted a 5-year longitudinal study with 115 men and women aged 60-84 and concluded that the risk of total and appendicular skeletal muscle mass loss was 1.29 times higher per unit of increase in IL-6 (pg/ml) and 1.28 times higher per unit of increase in high-sensitivity $\mathrm{C}$-reactive protein (CRP) $(\mathrm{mg} / \mathrm{l})$.

Recently, studies have used high body fat combined with low skeletal muscle mass to define sarcopenic obesity [10]. Yang et al. [11] performed a cross-sectional study in 844 people aged 65 years old living in the community and compared the inflammatory response among sarcopenic, obese, sarcopenic obese, and non-sarcopenic or non-obese individuals and observed no difference in the serum levels of IL- 6 and TNF- $\alpha$ between groups. However, CRP was higher among the obese and sarcopenic obese subjects. In addition, Zoico et al. [12] investigated the relationship between different muscle qualities by conducting muscle biopsies and comparing these with the body composition and inflammation in 16 sarcopenic men (aged between 58 and 80 years old) and demonstrated that the aging of skeletal muscle mass (SMM) is associated not only with muscle atrophy but also with adipose tissue, which worsens the metabolic profile.

Additionally, studies have shown positive correlations between the inflammatory profile, adiposity, metabolic alterations, and arterial properties, such as thickness and stiffness in the elderly $[13,14]$; therefore, microvascular damage correlated with stiffer artery may be the link between adiposity, inflammation, and systemic vascular damage. Furthermore, despite sarcopenic people presenting low muscle mass and strength, it is not clear whether the inflammatory and metabolic profile is more related to low lean mass or high fat mass in sarcopenic and non-sarcopenic people.

Thus, the objective of this study was to verify the differences in the inflammatory and metabolic response of sarcopenic and non-sarcopenic overfat elderly and the relationship between these markers, body composition, and strength in this population.

\section{Method}

\section{Participants}

This cross-sectional study was conducted in May 2015 in the city of Presidente Prudente (approximately 210,000 inhabitants), located in the western region of the state of São Paulo, Brazil. The participants were chosen through convenience sampling. A total of 307 adults of both genders, aged 60 years and older from the abovementioned city, were invited to participate in a previous cohort study, investigating elderly people and their relationship with physical activity, over 24 months. This study was advertised in the local media, and the individuals voluntarily presented themselves at the institution (Fig. 1).

The exclusion criteria for the current study were: inability to walk, being bedridden, and using pacemakers. The participants were informed regarding the study objectives and data collection methodology. Only individuals who signed the informed consent form were allowed to join the study. All protocols were reviewed and approved by the Research Ethics Committee of the São Paulo State University (Process $\mathrm{n}^{\mathrm{o}}:$ 15995113.8.0000.5402).

\section{Body composition assessment}

Initially, the body composition was assessed using a lunar dual-energy X-ray absorptiometry (DXA) scanner (model DPX-MD, software 4.7, General Electric Healthcare, Lunar DPX-NT; England). The percent body fat as DXA measurements from the 1999 to 2004 National Health and Nutrition Examination Survey (NHANES) [15] with body fat cutoffs from Lohman et al. [16] were used to determine the overfat condition.

For classification of sarcopenic, the appendicular lean soft tissue (ALST) was also determined by DXA. The ALST index was calculated as the ratio of ALST and height to the square (ALSTi), and then the z-score was calculated as the number of SD units from the sample ALSTi mean after normalization of the variable $(Z=[$ value - mean $] / S D)$, according to gender. Once the $z$ score was calculated, all the elderly, regardless of gender, were organized from the highest to the lowest value. After this procedure, the elderly with the 40 highest and 40 lowest $z$ score values were invited to participate in an intervention program involving resistance training. Of these, the 60 elderly who agreed to participate in the program were divided into a group of 30 sarcopenic individuals and 30 non-sarcopenic overfat individuals.

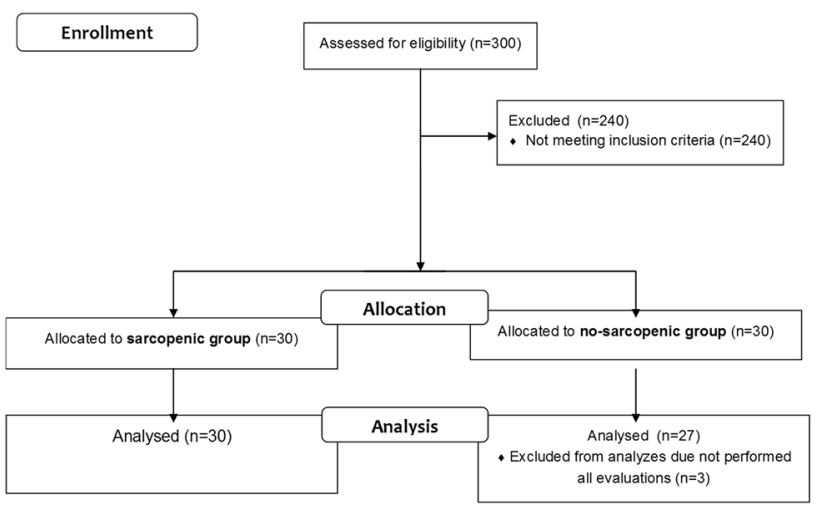

Fig. 1 Flow chart of the study $(n=57)$ 
Body mass, intra- and extracellular water, fat mass, musculoskeletal mass and fat and bone free mass from upper and lower limbs and trunk was measured with octopolar bioelectrical impedance equipment InBody 720 (Biospace, Seoul, Korea). Frequencies of 1, 5, 50, 250, 500 and $1000 \mathrm{kHz}$ was emitted and received from the contact of the subject heels and soles and thumbs and palms with the equipment. The minimum clothing was recommended to perform the measurement, according equipment manufacturer's recommendations: not eating and exercising before the test, urinate before the test and standing for $5 \mathrm{~min}$ before testing. Tests were performed indoors, at a controlled temperature between 20 and $25^{\circ} \mathrm{C}$.

\section{Strength test procedures}

Handgrip was measured on the dominant side, using an electronic hand dynamometer (model: EH101, Camry, China), with a precision of $0.1 \mathrm{~kg}$. To obtain the best performance, the dynamometer was adjusted so that it fitted comfortably to the subject's hand size. Participants, while seated with their elbow bent at an angle of $90^{\circ}$, were instructed to grip the dynamometer with maximum strength with the dynamometer facing outwards from the body. Three trials were performed, with a rest period of at least $1 \mathrm{~min}$ between trials. The highest value was considered as handgrip strength and the relative strength was obtained by dividing arm strength by skeletal muscle mass.

\section{Blood sampling and analyses}

Blood samples were collected after a 12-h fast, in the morning. The blood samples $(15 \mathrm{ml})$ were immediately allocated into two $5 \mathrm{ml}$ vacutainer tubes (Becton Dickinson, BD, Juiz de Fora, MG, Brazil) containing EDTA for plasma separation and one $5 \mathrm{ml}$ dry vacutainer tube for serum separation. The tubes were centrifuged at $3500 \mathrm{~g}$ for $15 \mathrm{~min}$ at $4{ }^{\circ} \mathrm{C}$, and plasma and serum samples were stored at $-20{ }^{\circ} \mathrm{C}$ until analysis. PAI-1 (ng/ml), leptin $(\mathrm{ng} / \mathrm{ml})$, and adiponectin $(\mu \mathrm{g} / \mathrm{ml})$ were assessed in serum samples using ELISA commercial kits (R\&D Systems, 614 McKinley Place NE, Minneapolis, MN 55413, USA). Glucose (mg/dl), total cholesterol (mg/ $\mathrm{dl})$, triacylglycerol (mg/dl), and HDL (mg/dl) were assessed in plasma samples using commercial kits (Labtest ${ }^{\circledR}$, São Paulo, Brazil). Cortisol was assessed using ELISA commercial kits (Monobind Inc. 100 North Point Drive, Lake Forest, CA 92630 USA). Interleukins (IL-6, IL-10 pg/ml) and TNF- $\alpha(\mathrm{pg} / \mathrm{ml})$ cytokines were assessed in serum samples using ELISA commercial kits (affimetrix/eBioscience, Ambriex S/A, São Paulo Brazil). To eliminate inter-assay variance, all samples were analyzed in identical runs, resulting in an intra-assay variance of $<7 \%$.

\section{Data analysis}

Initially, data distribution was checked using the Kolmogorov-Smirnov test and, based on the dataset, parametric statistics were performed and the data are described as mean and standard deviation. The independent $t$ test was used to verify the differences between groups in the body composition and performance. ANCOVA was performed to compare the metabolic and inflammatory profile, including skeletal muscle mass, fat mass, and the interaction term of both to see whether the role of selected variables differs between lean and overfat subjects with or without sarcopenia. The Pearson correlation $(r)$ was used to analyze the relationship between variables. Statistical analysis was performed using the SPSS statistical package version 17.0 (SPSS, Inc. Chicago, IL, USA) software and the level of statistical significance was set at $5 \%$.

\section{Results}

Table 1 presents the comparisons of body composition and performance values for the sarcopenic and non-sarcopenic overfat subjects.
Table 1 Comparison between age, height, weight, total and appendicular body composition and performance in sarcopenic and non-sarcopenic overfat subjects

\begin{tabular}{lccc}
\hline Variables & Non-sarcopenic $(n=27)$ & Sarcopenic $(n=30)$ & $p$ \\
\hline Body composition & & & $<\mathbf{0 . 0 0 1}$ \\
Fat mass $(\mathrm{kg})$ & $35.6 \pm 10.9$ & $20.4 \pm 6.2$ & $<\mathbf{0 . 0 0 1}$ \\
Visceral fat $\left(\mathrm{cm}^{2}\right)$ & $139.4 \pm 34.9$ & $90.8 \pm 24.0$ & $<\mathbf{0 . 0 0 1}$ \\
Arm lean mass $(\mathrm{kg})$ & $6.0 \pm 1.4$ & $3.8 \pm 1.0$ & $<\mathbf{0 . 0 0 1}$ \\
Leg lean mass $(\mathrm{kg})$ & $13.9 \pm 2.7$ & $10.6 \pm 2.3$ & $<\mathbf{0 . 0 0 1}$ \\
SMM $(\mathrm{Kg})$ & $27.7 \pm 5.3$ & $20.2 \pm 3.9$ & \\
Performance & & & 0.085 \\
Arm strength $(\mathrm{kg})$ & $28.5 \pm 9.7$ & $23.9 \pm 6.2$ & $\mathbf{0 . 0 1 4}$ \\
Relative strength & $4.9 \pm 1.4$ & $6.1 \pm 1.3$ & \\
\hline
\end{tabular}

SMM skeletal muscle mass 
It can be observed that the sarcopenic participants presented lower values of fat mass, visceral fat, appendicular muscle mass, and skeletal muscle mass when compared to the non-sarcopenic overfat subjects.

In relation to strength, there was a statistically significant difference between relative strength but no difference was observed in arm strength.

Table 2 presents the comparisons of metabolic and inflammatory profile between sarcopenic and non-sarcopenic overfat subjects.

When comparing the inflammatory profile, the sarcopenic group presented greater adiponectin concentration $(F=5.816, p=0.019)$, adiponectin/fat mass ratio $(F=20.480, p<0.001)$, adiponectin/visceral fat $(F=18.054$, $p<0.001)$, and higher PAI- $1(F=5.817, p=0.019)$ than non-sarcopenic overfat elderly. After adjusting the metabolic profile by skeletal muscle mass the significant differences between groups were maintained $(p<0.05)$ but there were no significant differences between groups when adjusting by fat mass, despite a tendency to a significant difference for adiponectin concentration $(p=0.06)$. In addition, after adjusting leptin by fat mass there was a statistically significant lower concentration in the sarcopenic compared to nonsarcopenic overfat elderly.

For TNF, IL-6, IL-8, IL-10, and metabolic profile (glucose, triacylglycerol, total cholesterol, and HDL-c) there were no significant differences between groups.

Table 3 presents the relationship between inflammatory and metabolic response, body composition, and strength in both groups studied.

Table 2 Comparison between sarcopenic and non-sarcopenic overfat subjects in the inflammatory and metabolic profile

\begin{tabular}{|c|c|c|c|c|c|c|}
\hline Variables & Non-sarcopenic $(n=27)$ & Sarcopenic $(n=30)$ & $p$ & $\begin{array}{l}p \\
\text { Adjusted by SSM }\end{array}$ & $\begin{array}{l}p \\
\text { Adjusted by } \\
\text { fat mass }\end{array}$ & $\begin{array}{l}p \\
\text { Adjusted by } \\
\text { SSM and fat } \\
\text { mass }\end{array}$ \\
\hline Glucose (mg/dl) & $96.3 \pm 21.4$ & $91.8 \pm 18.4$ & 0.402 & 0.717 & 0.790 & 0.682 \\
\hline Triacylglycerol (mg/dl) & $149.8 \pm 86.5$ & $156.1 \pm 81.5$ & 0.778 & 0.934 & 0.154 & 0.379 \\
\hline Chol (mg/dl) & $187.5 \pm 92.0$ & $204.4 \pm 79.3$ & 0.501 & 0.393 & 0.759 & 0.997 \\
\hline HDL-c (mg/dl) & $70.9 \pm 116.3$ & $62.2 \pm 61.5$ & 0.746 & 0.941 & 0.933 & 0.735 \\
\hline Cortisol $(\mu \mathrm{g} / \mathrm{ml})$ & $15.7 \pm 6.3$ & $13.6 \pm 6.5$ & 0.213 & 0.386 & 0.067 & 0.228 \\
\hline Leptin (ng/ml) & $221.4 \pm 149.9$ & $210.4 \pm 264.6$ & 0.851 & 0.219 & 0.030 & 0.395 \\
\hline Adiponectin $(\mu \mathrm{g} / \mathrm{ml})$ & $12.3 \pm 6.9$ & $18.2 \pm 10.9$ & 0.019 & 0.036 & 0.057 & 0.145 \\
\hline Adiponectin/fat mass & $0.34 \pm 0.2$ & $0.99 \pm 0.67$ & $<0.001$ & 0.005 & 0.085 & 0.241 \\
\hline Adiponectin/visceral fat & $0.08 \pm 0.05$ & $0.22 \pm 0.1$ & $<0.001$ & 0.015 & 0.115 & 0.389 \\
\hline PAI-1 (ng/ml) & $36.8 \pm 20.7$ & $54.5 \pm 32.8$ & 0.019 & 0.036 & 0.057 & 0.145 \\
\hline TNF- $\alpha(p g / m l)$ & $39.4 \pm 38.8$ & $39.1 \pm 37.3$ & 0.977 & 0.697 & 0.271 & 0.230 \\
\hline IL-6 (pg/ml) & $11.4 \pm 30.4$ & $3.5 \pm 5.0$ & 0.174 & 0.352 & 0.675 & 0.774 \\
\hline IL-8 (pg/ml) & $31.3 \pm 65.0$ & $19.5 \pm 39.0$ & 0.406 & 0.121 & 0.941 & 0.398 \\
\hline IL-10 (pg/ml) & $3.0 \pm 5.3$ & $1.8 \pm 1.4$ & 0.236 & 0.478 & 0.987 & 0.896 \\
\hline
\end{tabular}

Chol total cholesterol, PAI-1 Plasminogen activator inhibitor-1, TNF- $\alpha$ tumor necrosis factor alpha, $I L-6$ Interleukin-6, $I L-8$ Interleukin-8, $I L-10$ Interleukin-10

Table 3 Correlation between inflammatory and metabolic response, body composition and strength in sarcopenic and non-sarcopenic overfat subjects

\begin{tabular}{llllllll}
\hline Variables & Group & Fat mass $(\mathrm{kg})$ & Visceral fat $\left(\mathrm{cm}^{2}\right)$ & $\begin{array}{l}\text { Arm lean } \\
\text { mass }(\mathrm{kg})\end{array}$ & $\begin{array}{l}\text { Leg lean } \\
\text { mass }(\mathrm{kg})\end{array}$ & SMM $(\mathrm{kg})$ & Arm strength $(\mathrm{kg})$ \\
\hline Leptin & Non-sarcopenic & $\mathbf{0 . 4 3}^{*}$ & 0.27 & -0.33 & -0.38 & -0.38 & -0.06 \\
& sarcopenic & $\mathbf{0 . 6 1}^{* *}$ & $\mathbf{0 . 5 3}^{* *}$ & -0.13 & -0.21 & -0.21 & -0.32 \\
\multirow{2}{*}{ Adiponectin } & Non-sarcopenic & -0.21 & -0.31 & -0.40 & -0.33 & -0.37 & -0.14 \\
& Sarcopenic & -0.07 & -0.01 & 0.01 & 0.11 & 0.04 & 0.26 \\
\multirow{2}{*}{ PAI-1 } & Non-sarcopenic & -0.21 & -0.31 & -0.40 & -0.33 & -0.37 & -0.14 \\
& Sarcopenic & -0.07 & -0.01 & 0.01 & 0.11 & 0.04 & 0.26 \\
\hline
\end{tabular}

SMM skeletal muscle mass, PAI-1 Plasminogen activator inhibitor-1

$* p<0.05 ; * * p=0.001$ 
There was a moderate positive correlation between leptin and fat mass in the non-sarcopenic $(r=0.43, p=0.034)$ and sarcopenic groups $(r=0.61, p=0.001)$ and there were moderate positive correlations between leptin and visceral fat in the sarcopenic elderly $(r=0.53, p=0.004)$. No relationship was observed between adiponectin and PAI-1 concentration with body composition and strength in either group investigated.

\section{Discussion}

The main findings of this study were that the sarcopenic elderly presented a higher adiponectin/adiposity tissue ratio and lower PAI-1 than non-sarcopenic overfat elderly. Furthermore, fat mass, but not skeletal muscle mass, seems to change these responses. In addition, there were positive relationships between leptin, and total and visceral fat in both the sarcopenic and non-sarcopenic overfat elderly, although there was no correlation with skeletal muscle mass.

The higher adiponectin concentration and lower PAI-1 in sarcopenic people may be a consequence of the low fat mass in this group. Previous studies on nutritionally induced weight loss [17], gastric surgery [18], and exercise interventions demonstrated an increase in adiponectin [19]. Fatouros et al. [20] conducted a 1-year randomized, controlled trial that analyzed adiponectin responses after different intensities of exercise in 50 inactive men (65-78 years old) - both after exercise training and detraining - and observed that the percentage increase in adiponectin after training was associated with skinfold sum changes $(r=-0.64 ; p=0.001)$. Hsieh et al. [21] found that adiponectin levels increased with the reduction in weight, percentage of fat mass, and waist circumference in patients with type 2 diabetes.

Obesity, primarily visceral fat, also contributes significantly to increased plasma PAI-1, IL-6 levels, and low chronic inflammation, resulting in a higher risk of atherosclerosis and is linked to insulin resistance, impaired glucose tolerance, and elevated diabetes risk and metabolic syndrome [22], however, inhibition of PAI-1 in adipocytes protects against insulin resistance by promoting glucose uptake and adipocyte differentiation via increased PPAR $\gamma$ expression [23]. Studies have demonstrated that PAI-1 is overexpressed in adipose tissue. On the other hand, fat loss is associated with lower PAI-1 [24, 25].

In relation to muscle mass, the aging process is associated with the continuous transactivation of NF- $\mathrm{KB}$-dependent genes, resulting in many pro-inflammatory markers (TNF$\alpha$, IL-6, IL-10) [26]. Furthermore, in advanced age, reactive oxygen species (ROS) can stimulate signaling pathways, resulting in increased NF- $\mathrm{KB}$ activity and chronic inflammation [27]. In sarcopenic people, low muscle mass can occur via induction of the ubiquitin-proteasome (UbP) pathway and up-regulation of MuRF1 and atrogin-1 gene expression that modulates muscle wasting [28], which, once again increases TNF- $\alpha$ expression and implicates its function as a modifier of MuRF1 and atrogin-1 expression in older muscle [29].

Several studies in the literature demonstrate the relationship between these markers, body composition, and strength [7-9, 30, 31], however, in the present study there was a significant moderate-strong correlation in both sarcopenic and non-sarcopenic elderly only between leptin and total and visceral fat. Our findings are consistent in parts with the results from Lubkowska and colleagues [32] who correlated adiponectin and leptin concentrations with body fat distribution among obese subjects (23-54 years old) and found a positive correlation between leptin, body weight, BMI, and the waist-to-hip ratio. However, in Lubkowska's study, there was a significant negative correlation between adiponectin and total lean mass and skeletal muscle mass. High levels of inflammatory markers were correlated to a fast decline in functionality or a limitation in physical performance [33], given that TNF- $\alpha$ presented the strongest association with the decline in muscle mass and strength [34], however, the study by Legrand et al. [35], as well as our study, did not observe a relationship between inflammatory response and strength. On the other hand, it is known that muscle mass and strength do not decline similarly, thus, longitudinal studies are needed to better elucidate this relationship.

\section{Limitations}

The limitations of this study need to be considered when interpreting the findings. The cross-sectional design does not allow any inference of cause and effect and there was no nutritional control. Furthermore, we recommend future longitudinal studies and comparisons with sarcopenic overfat subjects.

\section{Conclusions}

In conclusion, the present investigation demonstrated that non-sarcopenic overfat elderly presented lower anti-inflammatory and anti-atherogenic responses than sarcopenic elderly. Furthermore, fat mass, but not skeletal muscle mass, seems to change these responses.

Acknowledgements The authors would like to thank CNPq (Conselho Nacional de Desenvolvimento Científico e Tecnológico) for the financial support (Edital Universal Proc. No. 483966/2013-4).

Funding This study was funded by the Edital Universal Proc. No. 483966/2013-4. 


\section{Compliance with ethical standards}

Conflict of interest The authors declare that they have no conflicts of interest.

Ethical approval All procedures performed were in accordance with the ethical standards of the institutional and/or national research committee and with the 1964 Helsinki declaration and its later amendments or comparable ethical standards. The study was reviewed and approved by the Research Ethics Committee of the São Paulo State University (Protocol no: 15995113.8.0000.5402).

Informed consent The present study complied with ethical standards and informed consent was obtained from all individual participants included in the study.

\section{References}

1. Bonnefoy M, Gilbert T (2015) Body composition and comorbidity in the elderly. Geriatrie et psychologie neuropsychiatrie du vieillissement 13:29-36. https://doi.org/10.1684/pnv.2015.0528

2. Landi F, Calvani R, Cesari M et al (2015) Sarcopenia as the biological substrate of physical frailty. Clin Geriatr Med 31:367-374. https://doi.org/10.1016/j.cger.2015.04.005

3. Ali NA, O'Brien JM Jr, Hoffmann SP et al (2008) Acquired weakness, handgrip strength, and mortality in critically ill patients. Am J Respir Crit Care Med 178:261-268. https://doi.org/10.1164/ rccm.200712-1829OC

4. Pedersen BK, Febbraio M (2005) Muscle-derived interleukin-6-a possible link between skeletal muscle, adipose tissue, liver, and brain. Brain Behav Immun 19:371-376. https://doi.org/10.1016/j. bbi.2005.04.008

5. Brandt C, Pedersen BK (2010) The role of exercise-induced myokines in muscle homeostasis and the defense against chronic diseases. J Biomed Biotechnol 2010:520258. https://doi. org/10.1155/2010/520258

6. Serrano AL, Baeza-Raja B, Perdiguero E et al (2008) Interleukin-6 is an essential regulator of satellite cell-mediated skeletal muscle hypertrophy. Cell Metab 7:33-44. https://doi.org/10.1016/j. cmet.2007.11.011

7. Visser M, Pahor M, Taaffe DR et al (2002) Relationship of interleukin-6 and tumor necrosis factor-alpha with muscle mass and muscle strength in elderly men and women: the Health ABC Study. J Gerontol Ser A Biol Sci Med Sci 57:M326-332

8. Aleman H, Esparza J, Ramirez FA et al (2011) Longitudinal evidence on the association between interleukin- 6 and C-reactive protein with the loss of total appendicular skeletal muscle in freeliving older men and women. Age Ageing 40:469-475. https://doi. org/10.1093/ageing/afr040

9. Schaap LA, Pluijm SM, Deeg DJ et al (2006) Inflammatory markers and loss of muscle mass (sarcopenia) and strength. Am J Med 119:526.e529-526.e517. https://doi.org/10.1016/j.amjme d.2005.10.049

10. Bouchard DR, Dionne IJ, Brochu M (2009) Sarcopenic/obesity and physical capacity in older men and women: data from the Nutrition as a Determinant of Successful Aging (NuAge)-the Quebec longitudinal Study. Obesity (Silver Spring Md) 17:20822088. https://doi.org/10.1038/oby.2009.109

11. Yang CW, Li CI, Li TC et al (2015) Correction: association of sarcopenic obesity with higher serum high-sensitivity C-reactive protein levels in Chinese older males-a community-based study (Taichung Community Health Study-Elderly, TCHS-E). PLoS One 10:e0136069. https://doi.org/10.1371/journal.pone.0136069
12. Zoico E, Corzato F, Bambace C et al (2013) Myosteatosis and myofibrosis: relationship with aging, inflammation and insulin resistance. Arch Gerontol Geriatr 57:411-416. https://doi. org/10.1016/j.archger.2013.06.001

13. Scuteri A, Orru M, Morrell C et al (2011) Independent and additive effects of cytokine patterns and the metabolic syndrome on arterial aging in the SardiNIA Study. Atherosclerosis 215:459464. https://doi.org/10.1016/j.atherosclerosis.2010.12.023

14. Scuteri A, Orru M, Morrell CH et al (2012) Associations of large artery structure and function with adiposity: effects of age, gender, and hypertension. The SardiNIA Study. Atherosclerosis 221:189 197. https://doi.org/10.1016/j.atherosclerosis.2011.11.045

15. Kelly TL, Wilson KE, Heymsfield SB (2009) Dual energy $\mathrm{X}$-Ray absorptiometry body composition reference values from NHANES. PloS One 4:e7038. https://doi.org/10.1371/journ al.pone.0007038

16. Lohman TG, Houtkooper L, Going SB (1997) Body fat measurement goes hightech: not all are created equal. ACSM'S Health Fit J 1:30-35

17. Zhu M, Miura J, Lu LX et al (2004) Circulating adiponectin levels increase in rats on caloric restriction: the potential for insulin sensitization. Exp Gerontol 39:1049-1059. https://doi.org/10.1016/j. exger.2004.03.024

18. Vidal J (2002) Updated review on the benefits of weight loss. Int J Obes Relat Metab Disord J Int Assoc Study Obes 26:S25-S28. https://doi.org/10.1038/sj.ijo.0802215

19. Hotta K, Funahashi T, Arita Y et al (2000) Plasma concentrations of a novel, adipose-specific protein, adiponectin, in type 2 diabetic patients. Arterioscler Thromb Vasc Biol 20:1595-1599

20. Fatouros IG, Tournis S, Leontsini D et al (2005) Leptin and adiponectin responses in overweight inactive elderly following resistance training and detraining are intensity related. J Clin Endocrinol Metab 90:5970-5977. https://doi.org/10.1210/jc.2005-0261

21. Hsieh CJ, Wang PW (2005) Effectiveness of weight loss in the elderly with type 2 diabetes mellitus. J Endocrinol Investig 28:973-977

22. Lalic K, Jotic A, Rajkovic N, Singh S et al (2015) Altered daytime fluctuation pattern of plasminogen activator inhibitor 1 in type 2 diabetes patients with coronary artery disease: a strong association with persistently elevated plasma insulin, increased insulin resistance, and abdominal obesity. Int J Endocrinol 2015:390185. https://doi.org/10.1155/2015/390185

23. Liang X, Kanjanabuch T, Mao SL et al (2006) Plasminogen activator inhibitor-1 modulates adipocyte differentiation. Am J Physiol Endocrinol Metab 290:E103-E113. https://doi.org/10.1152/ ajpendo.00605.2004

24. Chou YY, Sheu WH, Tang YJ et al (2009) Plasminogen activator inhibitor type 1 (PAI-1) is a valuable biomarker for predicting the metabolic syndrome (MS) in institutionalized elderly residents in Taiwan. Arch Gerontol Geriatr 49:S41-S45. https://doi. org/10.1016/s0167-4943(09)70012-3

25. Peverill RE, Teede HJ, Malan E et al (2007) Relationship of waist and hip circumference with coagulation and fibrinolysis in postmenopausal women. Clin Sci (Lond, Engl 1979) 113:383-391. https://doi.org/10.1042/cs20070084

26. Kim HK, Park HR, Lee JS et al (2007) Down-regulation of iNOS and TNF-alpha expression by kaempferol via NF-kappaB inactivation in aged rat gingival tissues. Biogerontology 8:399-408. https://doi.org/10.1007/s10522-007-9083-9

27. Li YP, Reid MB (2000) NF-kappaB mediates the protein loss induced by TNF-alpha in differentiated skeletal muscle myotubes. Am J Physiol Regul Integr Comp Physiol 279:R1165-R1170

28. Li YP, Chen Y, John J et al (2005) TNF-alpha acts via p38 MAPK to stimulate expression of the ubiquitin ligase atrogin1/MAFbx in skeletal muscle. FASEB J 19:362-370. https://doi.org/10.1096/ fj.04-2364com 
29. Clavel S, Coldefy AS, Kurkdjian E et al (2006) Atrophy-related ubiquitin ligases, atrogin-1 and MuRF1 are up-regulated in aged rat Tibialis Anterior muscle. Mech Ageing Dev 127:794-801. https://doi.org/10.1016/j.mad.2006.07.005

30. Biolo G, Di Girolamo FG, Breglia A et al (2015) Inverse relationship between "a body shape index" (ABSI) and fat-free mass in women and men: Insights into mechanisms of sarcopenic obesity. Clin Nutr (Edinb Scotl) 34:323-327. https://doi.org/10.1016/j. clnu.2014.03.015

31. Dutra MT, Avelar BP, Souza VC et al (2015) Relationship between sarcopenic obesity-related phenotypes and inflammatory markers in postmenopausal women. Clin Physiol Funct Imaging 37:205210. https://doi.org/10.1111/cpf.12287

32. Lubkowska A, Radecka A, Bryczkowska I et al (2015) Serum adiponectin and leptin concentrations in relation to body fat distribution, hematological indices and lipid profile in humans.
Int J Environ Res Public Health 12:11528-11548. https://doi. org/10.3390/ijerph120911528

33. Kilgour AH, Firth C, Harrison R et al (2013) Seropositivity for CMV and IL-6 levels are associated with grip strength and muscle size in the elderly. Immun Ageing I \& A 10:33. https://doi. org/10.1186/1742-4933-10-33

34. Schaap LA, Pluijm SM, Deeg DJ et al (2009) Higher inflammatory marker levels in older persons: associations with 5-year change in muscle mass and muscle strength. J Gerontol Ser A Biol Sci Med Sci 64:1183-1189. https://doi.org/10.1093/gerona/glp097

35. Legrand D, Adriaensen W, Vaes B et al (2013) The relationship between grip strength and muscle mass (MM), inflammatory biomarkers and physical performance in community-dwelling very old persons. Arch Gerontol Geriatr 57:345-351. https://doi. org/10.1016/j.archger.2013.06.003 